\title{
Tubercular Osteomyelitis of Talus with Staphylococcal Co infection
}

\section{Soraganvi P, Ramakanth R}

Department of Orthopaedics

PES Institute of Medical Science and Research

Kuppam, Chittor District- 517425

Andra Pradesh, India

\section{Corresponding Author}

Prasad Soraganvi

Department of Orthopaedics

PES Institute of Medical Science and Research

Kuppam, Chittor District- 517425

Andra Pradesh, India

\section{ABSTRACT}

Tuberculosis osteomyelitis of foot bones is uncommon and may evade the diagnosis for long time because of atypical presentation, paucibacillary nature and relatively normal picture on laboratory investigations. As tuberculosis of bone mimic like chronic osteomyelitis, clinical picture with confection by other organism lead to misdiagnosis. A case of tubercular osteomyelitis of talus reported here for its rarity and atypical presentation. Clinical suspicion and histological confirmation of diagnosis is recommended avoiding undue delay and for early chemotherapy.

\section{KEY WORDS}

Osteomyelitis, Staphylococcus, Talus, Tuberculosis

\section{Citation}

Soraganvi P, Ramakanth R. Tubercular Osteomyelitis of Talus with Staphylococcal Co infection. Kathmandu Univ Med J 2014;48(4):298-300.

\section{INTRODUCTION}

Tuberculosis is still a major health problem for both the developing and the developed countries. Isolated tuberculosis of talus is very rare and few cases are reported in literature. Tubercular involvement of the foot and ankle is difficult to diagnose, because of non-specific clinical and radiological findings. Most of the cases present with discharging sinus and swelling. The paucibacillary nature of discharge makes diagnosis more difficult. Many times invasive procedures become inevitable to establish the diagnosis. The case reported here presents the tuberculosis osteomyelitis of talus with Staphylococcus aureus coinfection in 24 year old male patient.

\section{CASE-REPORT}

Twenty four year aged man presented with history of swelling, pain and discharging wound in left ankle since 4 months. There was no history of trauma, fever, loss of weight or appetite. On examination his left ankle revealed swelling, tenderness and sinus discharging serosnguionus fluid (fig 1). Movements of ankle were restricted and painful. The laboratory studies revealed - haemoglobin -

12g/dl; total leucocytes count - 10600 cells/cu.mm with polymorphs $-68 \%$, lymphocytes- $29 \%$, eosinophils - $3 \%$; erythrocyte sedimentation rate $-37 \mathrm{~mm}$ in first hour. The routine chest roentgenogram was normal. Anteroposterior and lateral radiograph of ankle showed extensive irregular Iytic lesion of talus (fig 2). Talus was collapsed with decreased ankle joint space and irregular tibia articular surface, without the involvement of calcaneum. The ZiehlNeelsen stain of discharge taken from sinus tract was negative for acid fast bacilli but gram stain revealed gram positive cocci. The culture grew Staphylococcus aureus.

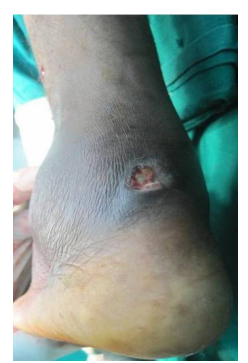

Figure 1. Discharging sinus seen on posterior view of ankle.

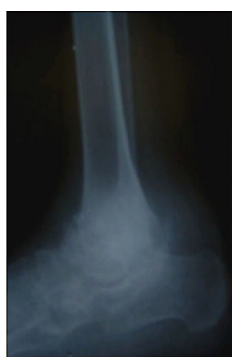

Figure 2. Lateral radiograph of ankle showing irregular lytic lesion with collapse of talus. Tibial articular surface is irregular with decreased joint space. 


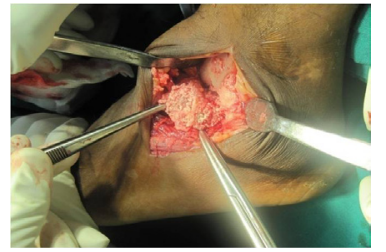

Figure 3. Operative pictures showing granulomatous tissue destroying talus and tibial articular surface.

By anterolateral approach ankle, talus and subtalar joint were exposed. Intra operatively inflammatory tissue comprising of numerous granulomatous excrescences were seen destroying talus and tibial articular surface (fig 3). Patient underwent talectomy followed by calcaneotibial arthrodesis which was fixed with Charlneys compression device (fig 4). For stability Steinman pin was inserted into tibia through calcaneum. Histological examination of biopsy sample showed tubercular granuloma with central caseating necrosis, compatible with tuberculosis of talus. Culture of biopsy material revealed growth of Staphylococcus aureus and Mycobacterium tuberculosis (Lowenstein Jensen medium). The patient received nine months of antitubercular chemotherapy consisting of three months of intensive therapy with four drugs (Isoniazid, Pyrazinamide, Ethambutol, Rifampicin) and continuous therapy for next six months with two drugs (Isoniazid, Rifampicin). Staphylococcus aureus was sensitive to Rifampicin hence no other antibiotic was deemed necessary. Healed sinus tract was noticed after six weeks. Charnyles compression device with Steinman pin were removed after eight weeks. Patient was advised non-weight bearing walking with below knee POP( Plaster of Paris) cast for four weeks followed by partial weight bearing walking with walking cast for four weeks. Full weight bearing walking was advised at 16 weeks following removal of POP cast. Patient recuperated well at end of chemotherapy. No evidence of recurrence has been detected at end of two year follow-up (fig 5 and fig 6).

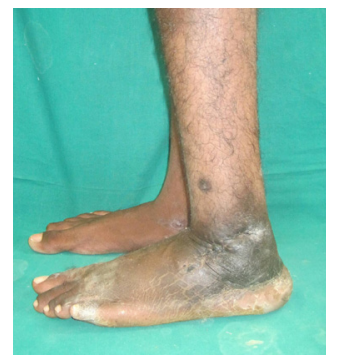

Figure 5. Follow-up clinical picture of patient showing satisfactory alignment of foot and ankle.

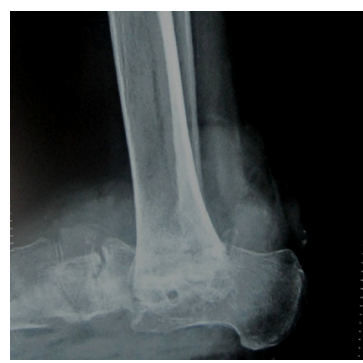

Figure 6. One year follow up radiograph showing satisfactory consolidation of calcaneotibial arthrodesis.

\section{DISCUSSION}

Tuberculosis remains a leading infection causing death world wide. ${ }^{1}$ The incidence of skeletal manifestation is only $1 \%$ to $3 \% .^{1,2}$ The ankle and foot are rarely affected and account for only $1 \%$ of all TB infections. ${ }^{3}$ Calcaneum is most commonly involved bone in foot. ${ }^{1,4,5}$ Tuberculosis of bone mimics clinical conditions like chronic osteomyelitis, madura mycosis, actinomycosis, multiple myeloma or secondary malignant deposits. ${ }^{6,7}$ Concomitant extra skeletal lesions or pulmonary tuberculosis are not seen in most of the patients. Lack of constitutional symptoms, confusing radiological features and relatively normal laboratory investigations lead to delay in diagnosis and misdiagnosis. ${ }^{1,2,8}$ The culture or smear may not be contributory to the diagnosis in majority of cases due to paucibacillary nature of disease when talus in involved. ${ }^{6}$ The confirmation of tuberculosis is done only by the identification of the bacillus from the local lesion or histological study of the tissue. This further underlines the need for a clinical suspicion of tuberculosis, so that early diagnosis and proper management can be carried out.

Only few cases of tuberculosis of talus have been reported in literature. ${ }^{9}$ Most of the cases of talus tubercular osteomyelitis reported earlier had late diagnosis due to lack of suspicion by treating surgeon making diagnosis difficult. The case presented here had concomitant infection with Staphylococcus aureus. The smear taken from discharge was negative for tubercular bacilli. This could be due to the paucibacillary nature of the disease. Such atypical presentation of skeletal tuberculosis is not uncommon in India where it is a major public health problem and hence tubercular osteomyelitis was considered in this patient. The confirmation of diagnosis was made only after histolopathogical findings which revealed tubercular granuloma with central caseating necrosis. Later isolation of tubercular bacilli in culture from tissue confirmed the diagnosis.

Atypical presentation, unusual site and paucibacillary nature of tubercular osteomyelitis of talus makes diagnosis extremely difficult. Such cases are easily misdiagnosed when concomitant infection with other organism is present. Thus a high index of clinical suspicion is mandatory. Clinical and radiologic features, along with histopathologic evidence of granulomatous pathology should be sufficient to initiate therapy.

Treatment of tuberculosis of ankle joint usually includes chemotheraphy, immobilization and non weight bearing protection. Subperiosteal cystic lesions treated by prolonged course of anti tubercular drugs. ${ }^{4}$ Debridement and curatage may be required in nonhealing lesion and when sequestrum is present. ${ }^{4}$ In local involvement of the talus bone, curettage and bone grafting with systemic chemotherapy is the treatment of choice. ${ }^{10}$ Joint involvement with minimal arthritic changes may be treated with arthroscopic synovectomy and debridement. Arthrodesis provides stable painless joint in painful ankle with advanced arthritis. ${ }^{4}$ We recommend talectomy and calcaneotibial arthrodesis when whole of talus is involved along with advanced arthritis of adjacent joint. Operative 
treatment along with chemotherapy affords painless stable joint with satisfactory weight bearing.

We report this case because of its rarity and tendency to misdiagnose easily when concomitant infection with Staphylococcus organism is present. We recommend

\section{REFERENCES}

1. Dhillon MS, Sharma S, Gill SS, Nagi ON. Tuberculosis of bones and joints of the foot: an analysis of 22 cases. Foot Ankle 1993;14(9): 505-13.

2. Anand A, Sood LK. Isolated tuberculosis of the talus without ankle and subtalar joints involvement. Med J Malaysia 2002; 57: 3713

3. Anderson JR, Ritchie AW, Scobie WG. Tuberculous osteitis of the talus. Tubercle 1979; 60: 115 18.10. Mittal R, upta V, Rastogi V. Tuberculosis of the foot. J Bone Joint Surg Br 1999; 81: 9971001

4. Mittal R, Gupta V, Rastogi S. Tuberculosis of the foot. J Bone Joint Surg 1999;81:997-1000.

5. Dhillon MS, Aggarwal S, Prabhakar S, Bachhal V. Tuberculosis of the foot: An osteolytic variety. Indian J Orthop 2012;46:206-11 clinical suspicion and histological investigation to avoid misdiagnosis. Awareness regarding atypical presentation of such an uncommon condition avoids undue delayed diagnosis. Management in the form of arthrodesis and chemotherapy results in good long term functional outcome.

6. Prakash A, Hira HS. Tuberculous osteomyelitis of sternum in a diabetic. Indian J Tuberc 2001;(48):35-6.

7. Tripathi AK, Gupta N, Khanna M, Ahmad R, Tripathi P. Tuberculosis presenting as osteolytic soft tissue swellings of skull in HIV positive patient : A case report. Indian J Tuberc 2007;(54):193-5.

8. Dhillon MS, Tuli SM. Osteoarticular tuberculosis of the foot and ankle. Foot Ankle Int 2001;22:679-86

9. Boussouga M, Tanane M, Bousselmame N, Lazrak K, Taobane H. Tuberculosis of the talus: a rare localization of Koch, $\mathrm{s}$ bacillus. Rev Chir Orthop Reparatrice Appar Mot 2002;88(5):522-5

10. Khan FA, Khoshhal K, Saadeddin M. Tuberculosis of talus and cuboid - a report of 2 children. Acta Orthop Scand 1999;70(6):637-9. 